\title{
Identification for Control of Multivariable Systems: Controller Validation and Experiment Design via LMIs
}

\author{
Märta Barenthin ${ }^{\mathrm{a}}$, Xavier Bombois ${ }^{\mathrm{b}}$, Håkan Hjalmarsson ${ }^{\mathrm{a}}$, Gérard Scorletti ${ }^{\mathrm{c}}$ \\ ${ }^{a}$ Automatic Control, School of Electrical Engineering, KTH, 10044 Stockholm, Sweden \\ ${ }^{\mathrm{b}}$ Delft Center for Systems and Control, Mekelweg 2, 2628 CD Delft, The Netherlands \\ ${ }^{\mathrm{c}}$ Laboratoire Ampère Ecole Centrale de Lyon, 36 avenue Guy de Collongue - 69134 Ecully Cedex, France
}

\begin{abstract}
This paper presents a new controller validation method for linear multivariable time-invariant models. Classical prediction error system identification methods deliver uncertainty regions which are nonstandard in the robust control literature. Our controller validation criterion computes an upper bound for the worst case performance, measured in terms of the $\mathcal{H}_{\infty}$-norm of a weighted closed loop transfer matrix, achieved by a given controller over all plants in such uncertainty sets. This upper bound on the worst case performance is computed via an LMI-based optimization problem and is deduced via the separation of graph framework. Our main technical contribution is to derive, within that framework, a very general parametrization for the set of multipliers corresponding to the nonstandard uncertainty regions resulting from PE identification of MIMO systems. The proposed approach also allows for iterative experiment design. The results of this paper are asymptotic in the data length and it is assumed that the model structure is flexible enough to capture the true system.
\end{abstract}

Key words: Identification for robust control; Experiment design; Closed loop system identification; Controller validation; Multivariable plants; Linear multivariable feedback; LMI optimization.

\section{Introduction}

In this paper, we develop robustness analysis tools which are appropriate for the particular uncertainty description delivered by the identification of a multi-input multi-output (MIMO) system. Additionally, we show how these tools can be used for experiment design.

Along with a model, an identification experiment in the prediction error (PE) framework also delivers an uncertainty region $D$ which contains the true system at some (user-chosen) probability level. Assuming that the model error is entirely due to variance effects, the identified uncertainty region $D$ is a set of parametrized transfer matrices $G(q, \theta)$ whose parameter vector $\theta$ is constrained to lie in an ellipsoid $U$ centered at the identified parameter vector $\hat{\theta}_{N}$ and whose size is determined by the covariance matrix $P\left(\theta_{o}\right)$ of $\sqrt{N} \hat{\theta}_{N}$, where $N$ is the number of input-output data collected on the true system. Such uncertainty structure is nonstandard in the classical robustness analysis literature. However, the paper [4] has

Email addresses: marta.barenthin@ee.kth.se (Märta Barenthin), x.j.a.bombois@tudelft.nl (Xavier Bombois), hakan.hjalmarsson@ee.kth.se (Håkan Hjalmarsson), gerard.scorletti@ec-lyon.fr (Gérard Scorletti). shown that, for the case of a single-input single-output (SISO) true system, robustness analysis tools can be derived for this particular uncertainty structure. These robustness analysis tools pertain to the validation of a controller $C$ designed from the identified model $G\left(q, \hat{\theta}_{N}\right)$. In particular, it is shown how to compute in an $\mathcal{H}_{\infty}$ framework the worst case performance achieved by the controller $C$ over the plants in $D$. If this worst case performance remains acceptable, the controller $C$ can then be applied to the true system $G_{o}$ since the performance achieved by $C$ over $G_{o}$ will be better than this worst case performance.

A larger uncertainty region $D$ implies worse control performance. Furthermore, the shape of $D$ depends on the experimental conditions under which the model and its uncertainty region have been identified. Based on this reasoning, research has been conducted in order to determine experimental conditions for which it is a-priori guaranteed that the obtained uncertainty region is small enough for the design of a robust controller achieving a given level of performance. This research has lead to the recent papers $[10,11,5]$ where the robustness conditions developed in [4] are explicitly used for the design of experimental conditions. Another approach for combining input design and robust control design is presented in $[2]$. 
The robustness conditions developed in [4] make extensive use of the fact that the true system is assumed SISO. As a consequence, these robustness tools cannot be extended as such to the case of MIMO systems ${ }^{1}$. Consequently, another approach is proposed in this paper to develop robustness analysis tools for the case of MIMO systems. For this purpose, the uncertain system is expressed as a linear fractional transformation (LFT) of a known transfer matrix $M$ and an uncertain part $\Delta$ only known to vary in a given set $\boldsymbol{\Delta}$. The robust performance properties of the uncertain system is then investigated based on the separation of graphs theorem $[15,8,16]$. Roughly speaking, the graph of a system is the set of its inputs/outputs. An extensive discussion on this topic can be found in $[8,16]$. If we can describe all the quadratic constraints satisfied by the graphs of all uncertainties $\Delta \in \boldsymbol{\Delta}$, then a necessary and sufficient condition guaranteeing the robust performance of the uncertain system is obtained from the separation of graphs theorem. Testing this necessary and sufficient condition is in general not tractable. However, a condition which is tractable but only sufficient can be deduced if we restrict attention to an explicit parametrization of the quadratic constraints satisfied by the graphs of all uncertainties $\Delta \in \Delta$. In [16], such a parametrization (called set of multipliers) is derived for some classical uncertainty sets encountered in the robustness analysis literature. However, the set $\boldsymbol{\Delta}$ corresponding to the MIMO systems in the uncertainty region $D$ is not at all classical. The set $\boldsymbol{\Delta}$ has indeed a very particular structure where the parameter vector $\theta$ of the systems in $D$ is repeated on the diagonal. To the best of our knowledge, such structure has never been considered in the literature. Consequently, our main technical contribution is to determine a (very general) parametrization for the set of multipliers corresponding to the set $\boldsymbol{\Delta}$.

Based on this set of multipliers, we develop an optimization problem involving linear matrix inequality (LMI) constraints which allow to compute an upper bound for the worst case performance achieved by a controller over the plants in $D$. We can only obtain an upper bound for the worst case performance since we apply the separation of graphs theorem restricting attention to a set of multipliers which is parametrized in an explicit way. However, this intrinsic conservatism is here strongly reduced by the choice of a very general set of multipliers (see [17] for an analysis of this conservatism reduction). Another contribution of this paper is to show how we can perform experiment design using the results on the validation of a controller for performance (worst case performance computation). The experiment design problem is also formulated as an LMI-based optimization problem.

Note that experiment design for MIMO systems is also treated in [6]. However, unlike the approach to experiment design proposed in the present paper, the approach presented in [6] is non-convex and the results can only

\footnotetext{
1 In [3], it is shown that the results in [4] can be extended to the case of multiple input single output systems, modulo the choice of a particular model structure. However, the extension of the result in [3] to MIMO systems is not possible.
}

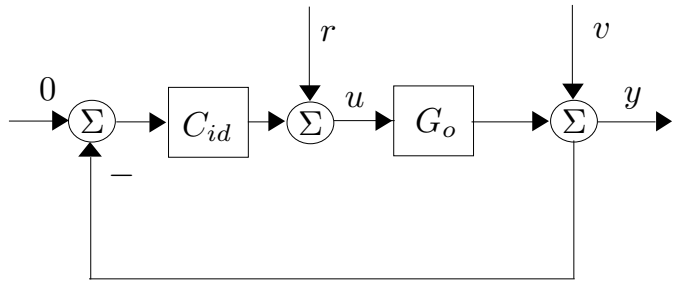

Fig. 1. The closed loop $\left[G_{o} C_{i d}\right]$ during identification.

be obtained if the identification is performed in a model structure which is linear in the parameter vector, such as Finite Impulse Response and Laguerre [20] model structures.

\section{Notation}

The transpose of $A$ is denoted $A^{T}$ and the complex conjugate transpose by $A^{*}$. For positive definite and positive semidefinite matrices the notation $A>0$ and $A \geq 0$ is used, respectively. The norm $\|\cdot\|_{\infty}$ is the $\mathcal{H}_{\infty}$-norm of the stable discrete transfer matrix $A\left(e^{j \omega}\right)$, i.e. $\|A\|_{\infty}=\sup _{\omega}\left\|A\left(e^{j \omega}\right)\right\|$ where $\|\cdot\|$ denotes the maximum singular value. The symbol $\otimes$ denotes the Kronecker product and $\star$ the Redheffer star product. Let $M$ denote a complex matrix partitioned as

$$
M=\left(\begin{array}{ll}
M_{11} & M_{12} \\
M_{21} & M_{22}
\end{array}\right) \in \mathbb{C}^{\left(p_{1}+p_{2}\right) \times\left(q_{1}+q_{2}\right)} .
$$

An (upper) LFT with respect to $\Delta \in \mathbb{C}^{q_{1} \times p_{1}}$ is defined as the map $\mathcal{F}(M, \bullet): \mathbb{C}^{q_{1} \times p_{1}} \mapsto \mathbb{C}^{p_{2} \times q_{2}}$ with $\mathcal{F}(M, \Delta)=$ $M_{22}+M_{21} \Delta\left(I-M_{11} \Delta\right)^{-1} M_{12}$, provided that the inverse $\left(I-M_{11} \Delta\right)^{-1}$ exists, see [23]. The time shift operator is given by $q$, i.e. $q r(t)=r(t+1), q^{-1} r(t)=r(t-1)$. Given an $m \times n$ matrix $X$, the operation $Y=\operatorname{vec}(X)$ produces the vector $Y$ of size $1 \times m n$ that contains the rows of the matrix $X$, stacked adjacent to each other.

\section{Prediction Error Identification Aspects}

We consider the following multivariable true system with output $y \in \mathbb{R}^{n_{y}}$ and input $u \in \mathbb{R}^{n_{u}}$,

$$
\begin{aligned}
\mathcal{S}: y(t) & =G_{o}(q) u(t)+v(t) \\
v(t) & =H_{o}(q) e(t),
\end{aligned}
$$

where $G_{o}(q)$ is a stable transfer matrix. Furthermore, $e(t) \in \mathbb{R}^{n_{y}}$ is white noise and $H_{o}(q)$ is a stable inversely stable monic transfer matrix. The power spectrum of the additive noise $v(t)$ is therefore given by $\Phi_{v}(\omega)=H_{o}\left(e^{j \omega}\right) \Lambda_{o} H_{o}^{*}\left(e^{j \omega}\right)$, where $\Lambda_{o}$ is the covariance of $e(t)$, i.e. $\mathrm{E}\left\{e(t) e^{T}(t)\right\}=\Lambda_{o}$. The true system will be identified within the following model structure chosen globally identifiable:

$$
\begin{aligned}
\mathcal{M}: y(t) & =G(q, \theta) u(t)+v(t) \\
v(t) & =H(q, \theta) e(t)
\end{aligned}
$$


where the vector $\theta \in \mathbb{R}^{n}$ represents the parameters to be identified. We assume that this model structure has been chosen in such a way that it is flexible enough to capture the true system i.e. there exists a parameter vector $\theta_{o}$ for which $G_{o}(q)=G\left(q, \theta_{o}\right)$ and $H_{o}(q)=H\left(q, \theta_{o}\right)$. The parameter estimate is then picked as

$\hat{\theta}_{N}=\underset{\theta}{\arg \min } \frac{1}{2 N} \sum_{t=1}^{N}(y(t)-\hat{y}(t, \theta))^{T} \Lambda_{o}^{-1}(y(t)-\hat{y}(t, \theta))$,

where the predictor $\hat{y}(t, \theta)$ is given by the stable filter

$$
\hat{y}(t, \theta)=H^{-1}(q, \theta) G(q, \theta) u(t)+\left[I-H^{-1}(q, \theta)\right] y(t),
$$

see $[19,13]$. Notice that (4) requires knowledge of the true noise covariance. One way of handling this contradiction in a practical situation is to estimate $\Lambda_{o}$ iteratively, see Section 15.2 in [13]. Both open loop and closed loop identification of the true system (2) is considered. For closed loop identification, the setup is represented in Figure 1 where $C_{i d}$ is the controller in the loop during identification and $r(t)$ is the excitation signal added to the loop in order to identify the system. In this configuration, the input and output signals used for the identification are given by:

$$
\begin{aligned}
& u(t)=\underbrace{S_{u}^{i d}(q) r(t)}_{=u_{r}(t)}-S_{u}^{i d}(q) C_{i d}(q) H_{o}(q) e(t) \\
& y(t)=\underbrace{S_{y}^{i d}(q) G_{o}(q) r(t)}_{=y_{r}(t)}+S_{y}^{i d}(q) H_{o}(q) e(t)
\end{aligned}
$$

where $S_{y}^{i d}=\left(I+G_{o} C_{i d}\right)^{-1}$ and $S_{u}^{i d}=\left(I+C_{i d} G_{o}\right)^{-1}$. For open loop identification, the excitation signal is directly applied via the input $u(t)$. Note that open loop identification is thus equivalent with closed loop identification for $C_{i d}=0$. Consequently, for the sake of brevity, we will in the sequel only consider closed loop identification since open loop identification is only a special case.

When the model is flexible enough to capture the true system, a very important property of the prediction error estimate $\hat{\theta}_{N}$ is that it is asymptotically normally distributed around the true parameter vector $\theta_{o}$ (i.e. $\hat{\theta}_{N} \sim$ $\left.\mathcal{N}\left(\theta_{o}, \frac{1}{N} P\left(\theta_{o}\right)\right)\right)$ as $N \rightarrow \infty$. This allows one to determine uncertainty regions for $\hat{\theta}_{N}$. In particular, $\hat{\theta}_{N}$ lies within the following ellipsoid in the parameter space:

$$
U=\left\{\theta:\left(\theta-\theta_{o}\right)^{T} P\left(\theta_{o}\right)^{-1}\left(\theta-\theta_{o}\right)<\frac{\chi}{N}\right\},
$$

with probability $\operatorname{Pr}\left(\chi^{2}(n) \leq \chi\right)$. The symbol $\chi^{2}(n)$ denotes the $\chi^{2}$-distribution with $n$ degrees of freedom. A corresponding uncertainty region for the model is thus:

$$
D=\{G(q, \theta) \mid \theta \in U\} .
$$

Note that $U$ and $P\left(\theta_{o}\right)$ depend on the true, and unknown, system. In controller validation, for a given model $\hat{\theta}_{N}$, we can construct a confidence region $U_{\hat{\theta}_{N}}$ for $\theta_{o}$, where

$$
U_{\hat{\theta}_{N}}=\left\{\theta:\left(\theta-\hat{\theta}_{N}\right)^{T} \hat{P}_{N}^{-1}\left(\theta-\hat{\theta}_{N}\right)<\chi\right\}
$$

with $\hat{P}_{N}$ an estimate of $\frac{1}{N} P\left(\theta_{o}\right)$ [13]. The true parameter vector $\theta_{o}$ is contained, to a certain probability, in $U_{\hat{\theta}_{N}}$. A corresponding confidence region for $G_{O}$ is thus:

$$
D_{\hat{\theta}_{N}}=\left\{G(q, \theta) \mid \theta \in U_{\hat{\theta}_{N}}\right\}
$$

In experiment design, the need for apriori system knowledge is a well known Achilles' heel [9,14]. One common approach to handle this issue in practice is by replacing $\theta_{o}$ by an initial estimate in (8) and then to use sequential procedures where the input design is altered on-line as more information becomes available, see e.g. [7].

\section{Controller Validation}

In the previous section, we showed how a model $G\left(\hat{\theta}_{N}\right)$ of the true system $G_{o}$ can be identified. Often, the purpose of this identified model $G\left(\hat{\theta}_{N}\right)$ is the design of a controller $C\left(\hat{\theta}_{N}\right)$ for the true system $G_{o}$. Since it has been designed from the identified model, the designed controller achieves of course satisfactory performance with this model. However, it is not guaranteed that the designed controller also achieves satisfactory performance with the true system. Since the true system is unknown, we cannot directly verify whether the controller is satisfactory with $G_{o}$. However, since we know that $G_{o}$ lies in the uncertainty region $D_{\hat{\theta}_{N}}$, we can verify whether the designed controller achieves satisfactory performance with all systems $G(\theta)$ in $D_{\hat{\theta}_{N}}$. If this is the case, the controller can be deemed validated since it is then guaranteed, with a probability chosen by the user, to achieve good performance with the true system. In this paper, we will measure the performance of a loop $[G C]$ via the largest singular value of a weighted closed loop transfer function. A general expression of this weighted closed loop transfer function is:

$$
J(G(\theta), C)=W_{l} F(G(\theta), C) W_{r},
$$

where $W_{l}$ and $W_{r}$ are weight matrices of appropriate dimensions and the matrix $F$ is one closed loop transfer function such as the sensitivity function $(I+G C)^{-1}$ or the complementary sensitivity function $G C(I+G C)^{-1}$. In fact, the only constraint on $F$ for the sequel is that it is a rational function of the system $G$ (or, in other words, an LFT in $G)^{2}$.

\footnotetext{
${ }^{2}$ Controller independent model quality measure such as $G-$ $G\left(\hat{\theta}_{N}\right)$ can therefore also be considered.
} 
Remark 1 Also the noise model $H(\theta)$ can be included in the performance function (12). The same LFT technique can be used in this case.

Based on the reasoning above, a very important quantity in order to validate the controller $C\left(\hat{\theta}_{N}\right)$ designed with the identified model is the worst case performance achieved over the plants in the confidence region $D_{\hat{\theta}_{N}}$ :

$$
\begin{aligned}
& \sigma_{W C}\left(C\left(e^{j \omega}, \hat{\theta}_{N}\right), D_{\hat{\theta}_{N}}\right)= \\
& =\max _{\theta \in U_{\hat{\theta}_{N}}}\left\|J\left(G\left(e^{j \omega}, \theta\right), C\left(e^{j \omega}, \hat{\theta}_{N}\right)\right)\right\| .
\end{aligned}
$$

The worst case performance (13) is thus a frequency function. Since $\sigma_{W C}\left(C\left(e^{j \omega}, \hat{\theta}_{N}\right), D_{\hat{\theta}_{N}}\right)$ is an upper bound of the performance $\left\|J\left(G_{o}\left(e^{j \omega}\right), C\left(e^{j \omega}, \hat{\theta}_{N}\right)\right)\right\|$ of the loop $\left[G_{o} C\left(\hat{\theta}_{N}\right)\right]$, the controller $C\left(\hat{\theta}_{N}\right)$ can be deemed validated if the worst case performance remains below a given threshold representing the required performance. We will show in the sequel that an upper bound for the worst case performance at each frequency can be obtained using LMI-based optimization.

The problem of computing, at each frequency $\omega$, the worst case performance achieved by a given controller $C\left(\hat{\theta}_{N}\right)$ over the plants in the confidence region $D_{\hat{\theta}_{N}}$ can be formulated equivalently as determining at each frequency the scalar $\gamma$ solving:

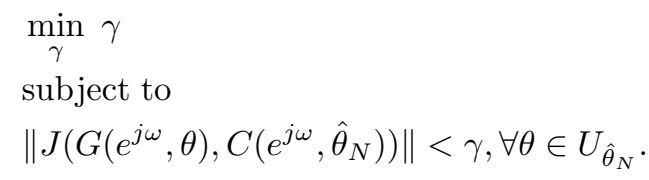

\section{A Tractable Formulation of the Worst Case Performance Problem in the Robustness Analysis Framework}

Computing the worst case performance at one frequency $\omega$ is thus equivalent to determining the smallest $\gamma$ such that the constraint (14) holds. The constraint (14) as such is not tractable. However, we will show in this section that if we solve an LMI-based optimization problem then the constraint (14) is satisfied. For this purpose, we will show that $J(G(\theta), C)$ can be expressed as an LFT in (a function of) $\theta$. (For an extensive discussion on LFT techniques, see i.e. [23]). This will allow us to use the following classical result of the robustness analysis literature that we present in the sequel in its general form (see Proposition 1) before particularizing it to the problem considered in the present paper. This result considers a transfer matrix $J$ of dimension $n_{y, J} \times n_{u, J}$ that can be expressed, for some given stable transfer matrix $M(q)$, as an LFT in a parametric uncertainty $\Delta$. The uncertainty $\Delta$ varies in a given set $\boldsymbol{\Delta}$, which contains the point $0:{ }^{3}$

$$
J(q, \Delta)=\mathcal{F}(M(q), \Delta) .
$$

In order to be able to use the result of Proposition 1 to verify a constraint similar to (14) for this uncertain transfer matrix $J$, it is required to associate to the parametric uncertainty set $\boldsymbol{\Delta}$ a so-called set of multipliers. The set $\mathcal{A}$ of multipliers used here is a set of affinely parametrized Hermitian matrices $A$ satisfying:

$$
\begin{aligned}
& A \in \mathcal{A} \Rightarrow \\
& \left(\begin{array}{l}
I \\
\Delta
\end{array}\right)^{*} \underbrace{\left(\begin{array}{ll}
A_{11} & A_{12} \\
A_{12}^{*} & A_{22}
\end{array}\right)}_{=A}\left(\begin{array}{l}
I \\
\Delta
\end{array}\right)>0, \forall \Delta \in \Delta
\end{aligned}
$$

The set $\mathcal{A}$ constitutes an affine parametrization of the quadratic constraints satisfied by the graph of all uncertainties $\Delta$.

Proposition 1 Consider a performance level $\gamma$ and a transfer matrix $J$ as defined in (15) for a stable $M$ and a parametric uncertainty $\Delta$ varying in a given set $\boldsymbol{\Delta}$ containing 0. Suppose furthermore that we have associated a set of multipliers to $\boldsymbol{\Delta}$. Then, at a given frequency $\omega$, a sufficient condition for

$$
\left\|J\left(e^{j \omega}, \Delta\right)\right\|<\gamma, \forall \Delta \in \Delta
$$

is the existence of a matrix $A$ in the set $\mathcal{A}$ such that

$$
\left(\begin{array}{c}
M\left(e^{j \omega}\right) \\
I
\end{array}\right) *\left(\begin{array}{cc|cc}
A_{11} & 0 & A_{12} & 0 \\
0 & I & 0 & 0 \\
\hline A_{12}^{*} & 0 & A_{22} & 0 \\
0 & 0 & 0 & -\gamma^{2} I
\end{array}\right)\left(M\left(e^{j \omega}\right)\right)<0
$$

PROOF. By the small gain theorem, expression (17) is equivalent to the fact that $\operatorname{det}\left(I-M\left(e^{j \omega}\right) \operatorname{diag}\left(\Delta, \Delta_{\text {ext }}\right)\right) \neq$ $0 \forall \Delta \in \Delta$ and for all complex matrices $\Delta_{\text {ext }}$ of dimension $n_{u, J} \times n_{y, J}$ such that $\left\|\Delta_{\text {ext }}\right\|<\frac{1}{\gamma}$, see e.g. [23,18]. The sufficient condition presented in the statement of the proposition is then a consequence of the separation of graphs theorem $[15,8,16]$.

Remark 2 It is important to stress that, in order to verify (17) at different frequencies $\omega$, the condition (18) can be verified with different matrices $A \in \mathcal{A}$.

\footnotetext{
${ }^{3}$ Note that the set $\boldsymbol{\Delta}$ must satisfy some additional minor assumptions which are classically met, see e.g. [16]. Note also that the result holds for any type of uncertainty, but we restrict our attention to parametric uncertainties since it is the case considered in this paper.
} 
The broader the parametrization of the matrices $A$ in the set of multipliers, the smaller the conservatism related to the non-necessary formulation of the condition (18). Proposition 1 formulates the constraint (17) as a convex feasibility problem. In order to use this proposition to formulate the constraint (14) also as a convex feasibility problem it is required to rewrite the functional $J\left(G\left(e^{j \omega}, \theta\right), C\left(e^{j \omega}, \hat{\theta}_{N}\right)\right)$ as an LFT in which $\theta$ represents the uncertainty and then to determine the set $\mathcal{A}$ of multipliers corresponding to the uncertainty involved in this LFT.

\subsection{LFT representation of $J\left(G(\theta), C\left(\hat{\theta}_{N}\right)\right)$}

As mentioned above, the functional $J$ must be formulated as an LFT. A first step towards this end is to note that $G(q, \theta)$ is a matrix of transfer functions for which each entry is a rational function of $\theta$. Consequently, each entry of $G(q, \theta)$ is an LFT in $\theta$. It is then a direct consequence of the properties of LFT functions that $G(\theta)$ is an LFT in

$$
m(\theta)=I_{\tilde{n}} \otimes \theta .
$$

where the value of $\tilde{n}$ depends on the particular parametrization (see the following example), but is always smaller than or equal to $n_{u} n_{y}$ (the number of entries of the matrix $G(q, \theta))$.

Example 1 An autoregressive exogeneous (ARX) model with 2 inputs/2 outputs is given by

$$
\begin{aligned}
& G(q, \theta)=\frac{1}{1+Z_{1}^{T} \theta}\left(\begin{array}{cc}
Z_{2}^{T} \theta & Z_{3}^{T} \theta \\
Z_{4}^{T} \theta & Z_{5}^{T} \theta
\end{array}\right), \\
& H(q, \theta)=\frac{1}{1+Z_{1}^{T} \theta} I_{2},
\end{aligned}
$$

where $\theta \in \mathbb{R}^{5}$ and $Z_{i}=q^{-1} e_{i}$ with $e_{i}$ defining the $i$ :th unit vector. The LFT description of $G(q, \theta)$ is $\mathcal{F}(X, m(\theta))$ with

$$
\begin{aligned}
X & =\left(\begin{array}{ll}
X_{11} & X_{12} \\
X_{21} & X_{22}
\end{array}\right), X_{11}=\left(\begin{array}{cc}
-Z_{1}^{T} & 0 \\
0 & -Z_{1}^{T}
\end{array}\right), X_{12}=I, \\
X_{21} & =\left(\begin{array}{ll}
Z_{2}^{T} & Z_{3}^{T} \\
Z_{4}^{T} & Z_{5}^{T}
\end{array}\right), X_{22}=0, m(\theta)=\left(\begin{array}{ll}
\theta & 0 \\
0 & \theta
\end{array}\right) .
\end{aligned}
$$

Consequently, for this model structure $\tilde{n}=2$.

Since $G(\theta)$ is an LFT in $m(\theta)$, it is also an LFT in $m(\delta \theta)$, where $\delta \theta=\theta-\hat{\theta}_{N}$, that is, $G(\theta)=\mathcal{F}\left(M_{G}, m(\delta \theta)\right)$ for some known $M_{G}$. Note that we consider $\delta \theta$ instead of $\theta$ to fulfill the requirement of Proposition 1 of a parametric uncertainty varying in a set containing 0 . Indeed, $\delta \theta$ is constrained to lie in $\tilde{U}$ where

$$
\tilde{U}=\left\{\delta \theta \mid(\delta \theta)^{T} \hat{P}_{N}^{-1} \delta \theta<\chi\right\}
$$

Now that we have shown that $G(\theta)$ is an LFT in $m(\delta \theta)$, we recall that $J(G(\theta), C)$ has been chosen as an LFT in $G(\theta)$ i.e. $J(G(\theta), C)=\mathcal{F}\left(M_{J}, G(\theta)\right)$ for some known $M_{J}$. Combining these two facts, we conclude that the transfer matrix $J(G(\theta), C)$ is also a LFT in $m(\delta \theta)$. Indeed,

$$
J\left(G(q, \theta), C\left(q, \hat{\theta}_{N}\right)\right)=\mathcal{F}(M(q), m(\delta \theta)),
$$

where $M(q)=M_{G}(q) \star M_{J}(q)$. Note that, as required by Proposition $1, M(q)$ is here stable since the loop $\left[C\left(q, \hat{\theta}_{N}\right) G\left(q, \hat{\theta}_{N}\right)\right]$ is stable.

\subsection{Set $\mathcal{A}$ of multipliers}

Given the LFT representation (23), in order to use Proposition 1 to obtain a convex sufficient condition for (14) to hold, we need to determine the set $\mathcal{A}$ of multipliers corresponding to the uncertainty $m(\delta \theta)$ present in the LFT (23). The matrices $A \in \mathcal{A}$ must therefore satisfy (16) for the parametric uncertainty $m(\delta \theta)$ with $\delta \theta \in \tilde{U}$ with $\tilde{U}$ defined in (22). This set of multipliers is given in the following proposition. Before giving this proposition, it is important to recall that the more general the parametrization of the matrices $A \in \mathcal{A}$ is, the less conservative the sufficient condition presented in Proposition 1 is for the verification of (17). Consequently, in Proposition 2, we present the most general affine parametrization we could find for the set of multipliers corresponding to the LFT (23).

Proposition 2 Consider the uncertainty $m(\delta \theta)$ with $\delta \theta \in \tilde{U}$ defined in (22). Define the parametrized set $\mathcal{A}$ of matrices $A$ as

$$
\mathcal{A}=\left\{A \mid A=\left(\begin{array}{ll}
A_{11} & A_{12} \\
A_{12}^{*} & A_{22}
\end{array}\right)\right\} .
$$

In this set the matrices $A_{11}, A_{12}$ and $A_{22}$ are affinely parametrized as follows:

$$
\begin{gathered}
A_{11}=A_{0}, \\
A_{12}=\left(\begin{array}{cccc}
j p_{11}^{T} & j p_{12}^{T} & \ldots & j p_{1 \tilde{n}}^{T} \\
j p_{12}^{T} & j p_{22}^{T} & \ldots & j p_{2 \tilde{n}}^{T} \\
\vdots & \vdots & \ddots & \vdots \\
j p_{1 \tilde{n}}^{T} & \ldots & \ldots & j p_{\tilde{n} \tilde{n}}^{T}
\end{array}\right)+\left(\begin{array}{cccc}
0 & \tilde{p}_{12}^{T} & \ldots & \tilde{p}_{1 \tilde{n}}^{T} \\
-\tilde{p}_{12}^{T} & 0 & \ldots & \tilde{p}_{2 \tilde{n}}^{T} \\
\vdots & \ddots & \ddots & \vdots \\
-\tilde{p}_{1 \tilde{n}}^{T} & \ldots & \ldots & 0
\end{array}\right), \\
A_{22}=-\left(A_{0} \otimes \frac{1}{\chi} \hat{P}_{N}^{-1}-j \tilde{A}+\tilde{B}\right) .
\end{gathered}
$$

The elements of this parametrization (i.e. $A_{0}, \tilde{A}, \tilde{B}, p_{l m}$ and $\left.\tilde{p}_{l m}, l=1,2, \ldots, \tilde{n} ; m=1,2, \ldots, \tilde{n}\right)$ can take any values provided that 
1) $A_{0}$ is a positive definite complex Hermitian matrix of dimension $\tilde{n} \times \tilde{n}$,

2) $\tilde{A} \in \mathbb{R}^{n \tilde{n} \times n \tilde{n}}$ belongs to the set

$$
\begin{array}{r}
\tilde{\mathbf{A}}=\left\{\begin{array}{c}
\left(\begin{array}{cccc}
L_{11} & L_{12} & \ldots & L_{1 \tilde{n}} \\
L_{12} & L_{22} & \ldots & L_{2 \tilde{n}} \\
\vdots & & \ddots & \vdots \\
L_{1 \tilde{n}} & L_{2 \tilde{n}} & \ldots & L_{\tilde{n} \tilde{n}}
\end{array}\right) \\
\mid L_{i j}=-L_{i j}^{T} \in \mathbb{R}^{n \times n}
\end{array}\right\}
\end{array}
$$

3) $\tilde{B} \in \mathbb{R}^{n \tilde{n} \times n \tilde{n}}$ belongs to the set

$$
\begin{aligned}
& \tilde{\mathbf{B}}=\left\{\left(\begin{array}{cccc}
0 & K_{12} & \ldots & K_{1 \tilde{n}} \\
-K_{12} & 0 & & \vdots \\
\vdots & & \ddots & K_{(\tilde{n}-1) \tilde{n}} \\
-K_{1 \tilde{n}} & \ldots & -K_{(\tilde{n}-1) \tilde{n}} & 0
\end{array}\right)\right. \\
& \left.\mid K_{i j}=-K_{i j}^{T} \in \mathbb{R}^{n \times n}\right\}
\end{aligned}
$$

4) $p_{l m}, \tilde{p}_{l m} \in \mathbb{R}^{n}, l=1,2, \ldots, \tilde{n}$ and $m=1,2, \ldots, \tilde{n}$.

Then for any $A \in \mathcal{A}$ we have

$$
\left(\begin{array}{c}
I \\
m(\delta \theta)
\end{array}\right)^{*} A\left(\begin{array}{c}
I \\
m(\delta \theta)
\end{array}\right)>0, \quad \forall \delta \theta \in \tilde{U}
$$

PROOF. First, notice that, for all $A_{12}, \tilde{A}$ and $\tilde{B}$ as defined in the statement of the proposition, we have:

$$
\begin{array}{r}
A_{12} m(\delta \theta)+(m(\delta \theta))^{T} A_{12}^{*}=0 \\
(m(\delta \theta))^{T} j \tilde{A} m(\delta \theta)=0 \\
(m(\delta \theta))^{T} \tilde{B} m(\delta \theta)=0 .
\end{array}
$$

Therefore, for every matrix $A \in \mathcal{A}$, we can write

$$
\begin{aligned}
& \left(\begin{array}{c}
I \\
m(\delta \theta)
\end{array}\right)^{*} A\left(\begin{array}{c}
I \\
m(\delta \theta)
\end{array}\right)= \\
& =\left(1-\delta \theta^{T}\left(\frac{1}{\chi} \hat{P}_{N}^{-1}\right) \delta \theta\right) A_{0},
\end{aligned}
$$

and we observe that this matrix is indeed positive definite when $\delta \theta \in \tilde{U}$ since $A_{0}>0$.

Remark 3 The determination of the set of multipliers corresponding to the uncertainty involved in the LFT (23) is the main technical contribution of this paper. Indeed, to our knowledge, such uncertainty structure has never been considered in the robustness analysis literature. An interesting feature of this set of multipliers is the skewsymmetric multipliers $\tilde{A}$ and $\tilde{B}$ in the diagonal block $A_{22}$. Indeed, skew-symmetric terms (such as $A_{12}$ here) are generally only present in the off-diagonal blocks.
Remark 4 The matrices $A \in \mathcal{A}$ are explicit functions of the inverse $\hat{P}_{N}^{-1}$ of the estimated covariance matrix. This is not an issue here since $\hat{P}_{N}$ is known. However, this observation will be important when we consider experiment design in Section 6.

\subsection{Sufficient convex formulation of constraint (14)}

Gathering the LFT representation (23), the set of multipliers defined in Proposition 2 and the result of Proposition 1, we can now formulate the constraint (14) as a convex feasibility problem involving LMI constraints.

Theorem 1 Consider the constraint (14) at a particular frequency $\omega$ and suppose that $\gamma$ is given. Then, the constraint (14) holds if there exists a matrix $A$ as parametrized in Proposition 2 such that the following LMI holds:

$$
\left(\begin{array}{c}
M\left(e^{j \omega}\right) \\
I
\end{array}\right) *\left(\begin{array}{cc|cc}
A_{11} & 0 & A_{12} & 0 \\
0 & I & 0 & 0 \\
\hline A_{12}^{*} & 0 & A_{22} & 0 \\
0 & 0 & 0 & -\gamma^{2} I
\end{array}\right)\left(\begin{array}{c}
M\left(e^{j \omega}\right) \\
I
\end{array}\right)<0
$$

for $M(q)$ as defined in (23).

PROOF. This theorem is a direct consequence of Proposition 1. Indeed, the functional $J$ is represented by the LFT (23) and the matrix $A$ as defined in Proposition 2 represent the set of multipliers for the uncertainty involved in (23). Note that (35) is indeed an LMI since the matrix $A$ is linearly parametrized in the elements $A_{0}$, $\tilde{A}, \tilde{B}, p_{l m}$ and $\tilde{p}_{l m}, l=1,2, \ldots, \tilde{n} ; m=1,2, \ldots, \tilde{n}$.

\subsection{Computing an upper bound for the worst case per-} formance

Observe that (35) is not only affine in the matrices $A_{11}$, $A_{12}, A_{22}$, but also in the variable $\gamma^{2}$. Based on this observation, we are now ready to derive an algorithm to compute a frequency wise upper bound for the worst case performance achieved by the designed controller $C\left(\hat{\theta}_{N}\right)$ over the plants in the confidence region $D_{\hat{\theta}_{N}}$.

Theorem 2 Consider, at a particular frequency $\omega$, the worst case performance $\sigma_{W C}\left(C\left(e^{j \omega}, \hat{\theta}_{N}\right), D_{\hat{\theta}_{N}}\right)$ achieved by a controller $C\left(\hat{\theta}_{N}\right)$ over the plants in a confidence region $D_{\hat{\theta}_{N}}$. An upper bound for $\sigma_{W C}\left(C\left(e^{j \omega}, \hat{\theta}_{N}\right), D_{\hat{\theta}_{N}}\right)$ can be obtained by solving the LMI-based optimization problem consisting of determining the smallest value $\gamma_{\text {opt }}^{2}$ of $\gamma^{2}$ for which there exists a matrix $A$ in the set $\mathcal{A}$ defined in (24) such that (35) holds. The upper bound on $\sigma_{W C}\left(C\left(e^{j \omega}, \hat{\theta}_{N}\right), D_{\hat{\theta}_{N}}\right)$ is then given by $\sqrt{\gamma_{o p t}^{2}}$. 
PROOF. The worst case performance at the frequency $\omega$ can be computed by finding the smallest $\gamma$ such that (14) holds. Consequently, this theorem is a direct consequence of Theorem 1.

Since Theorem 2 only delivers an upper bound for $\sigma_{W C}\left(C\left(e^{j \omega}, \hat{\theta}_{N}\right), D_{\hat{\theta}_{N}}\right)$, it may be important to check how conservative this upper bound is. Due to the very general parametrization of the set of multipliers, this conservatism should be limited (see [17]). However, in order to have a good idea of the conservatism, we will, besides the upper bound, also compute a lower bound for $\sigma_{W C}\left(C\left(e^{j \omega}, \hat{\theta}_{N}\right), D_{\hat{\theta}_{N}}\right)$. This can be done e.g. by gridding the uncertainty region $U_{\hat{\theta}_{N}}$ and take, at each $\omega$, the maximal value of $\left\|J\left(G\left(e^{j \omega}, \theta\right), C\left(e^{j \omega}, \hat{\theta}_{N}\right)\right)\right\|$ for the $\theta$ in this grid.

\section{Experiment Design}

Let us sum up what we have done until now. We consider the situation where a model $G\left(\hat{\theta}_{N}\right)$ of the true system $G_{o}$ has been deduced by an identification experiment (in open loop or closed loop). Based on this model, we have designed a controller $C\left(\hat{\theta}_{N}\right)$ for the true system. In order to verify whether this controller is satisfactory for the true system, we need to verify whether this controller achieves satisfactory performance with all plants in the constructed confidence region $D_{\hat{\theta}_{N}}$. In order to do that, we have shown in Theorem 2 how to compute an upper bound for the worst case performance achieved by the controller over the plants in $D_{\hat{\theta}_{N}}$. The validation of the controller can therefore be achieved by checking that this bound is at each frequency below some threshold representing the required performance.

It can happen, the uncertainty region $D_{\hat{\theta}_{N}}$ being too large, that the worst case performance cost is too large for the controller to be applied to the true system. A new identification has therefore to be performed in order to obtain a more accurate model. Assuming that the number $N$ of data in the second experiment is the same as in the initial experiment, the only method to reduce the size of the uncertainty region is to modify the power spectrum $\Phi_{r}(\omega)$ of the excitation signal $r(t)$. Indeed, the asymptotic covariance matrix $P\left(\theta_{o}\right)$ which defines the size of $U$ is uniquely influenced by $\Phi_{r}(\omega)$. In fact, $P^{-1}\left(\theta_{o}\right)$ is an affine function in $\Phi_{r}(\omega)[13,1,5]$ :

$$
P^{-1}\left(\theta_{o}\right)=R_{1}\left(\Phi_{r}(\omega), \theta_{o}\right)+R_{2}\left(\theta_{o}\right) .
$$

For the MIMO case, the matrices $R_{1}\left(\Phi_{r}(\omega), \theta_{o}\right)$ and $R_{2}\left(\theta_{o}\right)$ are given by the following proposition.

Proposition 3 The matrices $R_{1}\left(\Phi_{r}(\omega), \theta_{o}\right)$ and $R_{2}\left(\theta_{o}\right)$ in (36) are given by

$$
\begin{aligned}
& R_{1}\left(\Phi_{r}(\omega), \theta_{o}\right)= \\
& =\frac{1}{2 \pi} \int_{-\pi}^{\pi} \Gamma_{r}\left(e^{j \omega}, \theta_{o}\right)\left(\Lambda_{o}^{-1} \otimes \Phi_{r}\left(e^{j \omega}\right)\right) \Gamma_{r}^{*}\left(e^{j \omega}, \theta_{o}\right) d \omega \\
& R_{2}\left(\theta_{o}\right)=\frac{1}{2 \pi} \int_{-\pi}^{\pi} \Gamma_{e}\left(e^{j \omega}, \theta_{o}\right)\left(\Lambda_{o}^{-1} \otimes \Lambda_{o}\right) \Gamma_{e}^{*}\left(e^{j \omega}, \theta_{o}\right) d \omega
\end{aligned}
$$

where

$$
\Gamma_{r}(q, \theta)=\left(\begin{array}{c}
\operatorname{vec}\left(F_{r}^{1}(q, \theta)\right) \\
\operatorname{vec}\left(F_{r}^{2}(q, \theta)\right) \\
\vdots \\
\operatorname{vec}\left(F_{r}^{n}(q, \theta)\right)
\end{array}\right)
$$

$$
\Gamma_{e}(q, \theta)=\left(\begin{array}{c}
\operatorname{vec}\left(F_{e}^{1}(q, \theta)\right) \\
\operatorname{vec}\left(F_{e}^{2}(q, \theta)\right) \\
\vdots \\
\operatorname{vec}\left(F_{e}^{n}(q, \theta)\right)
\end{array}\right)
$$

$$
F_{r}^{i}(q, \theta)=H_{o}^{-1}(q) \frac{d G(q, \theta)}{d \theta_{i}} S_{u}^{i d}(q)
$$

$$
\begin{aligned}
F_{e}^{i}(q, \theta) & =H_{o}^{-1}(q)\left(\frac{d H(q, \theta)}{d \theta_{i}}-\right. \\
& \left.-\frac{d G(q, \theta)}{d \theta_{i}} S_{u}^{i d}(q) C_{i d}(q) H_{o}(q)\right), \\
i & =1, \ldots, n
\end{aligned}
$$

and $\theta_{i}$ defines the $i$ :th component of the vector $\theta$.

PROOF. See Appendix A.

In the sequel, we will present a way to optimally determine the spectrum $\Phi_{r}(\omega)$ for the second experiment. This experiment design procedure is an extension to the MIMO case of the previous work done in the SISO case (see e.g. $[12,11,5])$. An important step in this procedure is to parametrize the spectrum $\Phi_{r}(\omega)$. A common parametrization is as follows:

$$
\Phi_{r}(\omega)=\sum_{k=-m}^{m} c_{k} e^{-j \omega k}
$$

where $m$ is a user-choice and the matrices $c_{k} \in$ $\mathbb{R}^{n_{u} \times n_{u}}, k=-m, \ldots, m$, must be such that $c_{-k}=c_{k}^{T}$ 
and such that $\Phi_{r}(\omega) \geq 0, \forall \omega$. Using the Positive Real Lemma $[22,21]$, the constraint that $\Phi_{r}(\omega) \geq 0, \forall \omega$, can be recast into an LMI constraint on the matrices $c_{k}, k=-m, \ldots, m$, (see $\left.[12,11,5]\right)$. The matrices $c_{k}, \quad k=-m, \ldots, m$, in (44) entirely determine the spectrum $\Phi_{r}(\omega)$ and are the actual decision variables of the experiment design problem. In this aspect, it is important to note that any affine function of $\Phi_{r}(\omega)$ (such as e.g. $P^{-1}\left(\theta_{o}\right)$ ) is also an affine function of the frequency-independent matrices $c_{k}, k=-m, \ldots, m$, .

In this experiment design problem, our objective is to determine the matrices $c_{k}, k=-m, \ldots, m$, in (44) in such a way that the corresponding spectrum $\Phi_{r}(\omega)$ is the least disturbing spectrum ${ }^{4}$ which nevertheless guarantees that the covariance matrix $P\left(\theta_{o}\right)$ is small enough for (45) to hold:

$$
\begin{array}{r}
\left\|J\left(G\left(e^{j \omega}, \theta\right), C\left(e^{j \omega}, \hat{\theta}_{N}\right)\right)\right\|<\gamma_{\max }(\omega) \forall \omega, \\
\forall \theta \in U
\end{array}
$$

In $(45), \gamma_{\max }(\omega)$ is a given threshold representing the minimal admissible performance. Furthermore, $\hat{\theta}_{N}$ and $C\left(\hat{\theta}_{N}\right)$ are the ones corresponding to the experiment we want to design and are thus unknown at the very moment we design the experiment. They are therefore replaced by initial estimates, $c f$ Section 3. Reasonable initial estimates (see e.g. [5]) are those obtained in the initial experiment ${ }^{5}$. See also $[1]$.

The constraint (45) is an infinite-dimensional constraint since it must hold at each frequency. This constraint can nevertheless be made finite-dimensional by considering a finite frequency grid $\Omega$ instead of the whole frequency range. The corresponding optimization problem is thus made up of one constraint per frequency in the finite grid $\Omega$ (see [11]). Given the result of Theorem 1, this problem can be reformulated as: determine the matrices $c_{k}, k=-m, \ldots, m$, corresponding to the least costly spectrum for which we can still find, $\forall \omega \in \Omega$, a matrix $A(\omega) \in \mathcal{A}$ for which it holds that:

$$
\left(\begin{array}{c}
M\left(e^{j \omega}\right) \\
I
\end{array}\right)^{*}\left(\begin{array}{cc|cc}
A_{11}(\omega) & 0 & A_{12}(\omega) & 0 \\
0 & I & 0 & 0 \\
\hline A_{12}^{*}(\omega) & 0 & A_{22}(\omega) & 0 \\
0 & 0 & 0 & -\gamma_{\max }^{2}(\omega) I
\end{array}\right)\left(\begin{array}{c}
M\left(e^{j \omega}\right) \\
I
\end{array}\right)<0 .
$$

Here, we use the notation $A_{11}(\omega)$ etc. to stress that these matrices can be different at each frequency (see Remark 2). Note also that, in the parametrization of the

\footnotetext{
4 i.e. the one with smallest $\operatorname{Tr}\left(\int_{-\pi}^{\pi} \Phi_{u_{r}}(\omega)+\Phi_{y_{r}}(\omega) d \omega\right)$, where $u_{r}$ and $y_{r}$ are defined in (6)-(7). This expression is affine in $\Phi_{r}(\omega)$ and thus also in the matrices $c_{k}, k=$ $-m, \ldots, m$, defining $\Phi_{r}(\omega)$.

5 The parameter vector $\hat{\theta}_{N}$ identified in the first experiment is generally also used to approximate $\theta_{o}$ in (36).
}

set of multipliers $\mathcal{A}, \hat{P}_{N}^{-1}$ is replaced by $N P^{-1}\left(\theta_{o}\right)$. The constraint (46) at each $\omega \in \Omega$ is dependent on the decision variables $c_{k}, k=-m, \ldots, m$, via the parametrization of $A(\omega)$. Indeed, $A_{22}(\omega)$ is a function of $P^{-1}\left(\theta_{o}\right)$ and thus of the matrices $c_{k}, k=-m, \ldots, m$, via $(36)$ and (44). Even though $P^{-1}\left(\theta_{0}\right)$ is affine in the matrices $c_{k}, k=-m, \ldots, m$, defining $\Phi_{r}(\omega)$ (see above), the constraint (46) is not an LMI due to the product of $P^{-1}\left(\theta_{o}\right)$ and the multiplier $A_{0}(\omega)$ which is also a decision variable. The only solution is then to modify the set $\mathcal{A}$ of multipliers by fixing $A_{0}(\omega)$ to a constant matrix e.g. the identity matrix. For a fixed $A_{0}(\omega),(46)$ is an LMI in the matrices $c_{k}, k=-m, \ldots, m$, and these matrices can therefore be easily determined. Fixing $A_{0}(\omega)$ nevertheless increases conservatism since the set of multipliers is then less general and it is furthermore very unlikely that the chosen $A_{0}(\omega)$ is the optimal for the considered optimization problem. In order to improve the choice of $A_{0}(\omega)$, we propose the following iterative procedure inspired by the so called D-K iterations [23]. In a first step, we determine the matrices $c_{k}, k=-m, \ldots, m$, with a fixed $A_{0}(\omega)$ as already described. This delivers the matrices $c_{k \text {,int }}$. In a second step, we then parametrize the matrices $c_{k}, k=-m, \ldots, m$, as $c_{k}=\lambda c_{k, \text { int }}$ with $\lambda$ a to-be-optimized scalar and we then determine by dichotomy the smallest $\lambda$ for which we can find at each frequency $\omega \in \Omega$ a matrix $A(\omega) \in \mathcal{A}$ for which (46) holds, but this time with a free $A_{0}(\omega)((46)$ is indeed an LMI in $A_{0}$ for a fixed $\lambda i$.e. for fixed $\left.c_{k}, k=-m, \ldots, m\right)$. Denote $\forall \omega \in \Omega$ by $A_{0, \text { int }}(\omega)$ the value for $A_{0}(\omega)$ found with this optimal $\lambda$. Next we repeat the first step with $A_{0}(\omega)$ now fixed to $A_{0, \text { int }}(\omega)$, etc. Note that multiplying the matrices $c_{k, \text { int }}$ by a scalar $\lambda$ corresponds to multiplying the corresponding spectrum by $\lambda$, i.e. $\lambda \Phi_{r, \text { int }}(\omega)$.

Remark 5 For the case the goal of the experiment design is to reduce the duration of the experiment, we can by dichotomy find the smallest $N$ for which (46) holds $\forall \omega \in$ $\Omega$, for a given $\Phi_{r}(\omega)$.

\section{Numerical Illustration}

Consider the model in Example 1 with the true dynamics of the system defined by

$$
\theta_{o}=(-0.7558,0.4577,-0.2180,0.2180,-0.4577)^{T}
$$

and $\Lambda_{o}=0.2 I_{2}$. First a closed loop identification experiment with $N=1000$ data is performed. The controller in the loop is $C_{i d}=C_{\text {start }}$ where

$$
C_{\text {start }}(q)=\left(\begin{array}{cc}
\frac{Z^{T} \eta_{s, 1}}{Z^{T} \eta_{s, 3}} & \frac{Z^{T} \eta_{s, 2}}{Z^{T} \eta_{s, 3}} \\
-\frac{Z^{T} \eta_{s, 2}}{Z^{T} \eta_{s, 3}} & -\frac{Z^{T} \eta_{s, 1}}{Z^{T} \eta_{s, 3}}
\end{array}\right)
$$

Here $Z=\left(1 q^{-1} q^{-2} \ldots q^{-6}\right)^{T}$ and $\eta_{s, 1}, \eta_{s, 2}, \eta_{s, 3}$ are given in Appendix B. Furthermore, since the controller $C_{\text {start }}$ is of sufficient order, it is so that the closed loop 
identification can be performed consistently using the excitation of the noise $e(t)$ exclusively i.e. with $r(t)=0$. Therefore, we decide to collect $N=1000$ data in this particular situation. The following parameter estimate is obtained:

$$
\hat{\theta}_{N, 1}=(-0.7344,0.4039,-0.1706,0.2378,-0.4740)^{T} .
$$

Now the proposed controller validation procedure will be applied. The closed loop expression (12) is given by

$$
J\left(G(\theta), C_{1}\right)=W_{S}\left(I+G(\theta) C_{1}\right)^{-1}
$$

where $W_{S}(q)$ is a scalar weight transfer function whose inverse is plotted in Figure $3^{6}$ and $C_{1}=C_{1}\left(\hat{\theta}_{N, 1}\right)$ is a model based controller constructed using a 4 -block $\mathcal{H}_{\infty}$ design method where one of the objectives is to satisfy that the $\mathcal{H}_{\infty}$-norm of the right hand side of (50) is less than one. Here

$$
C_{1}(q)=\frac{1}{Z^{T} \eta_{1,5}}\left(\begin{array}{cc}
Z^{T} \eta_{1,1} & Z^{T} \eta_{1,2} \\
Z^{T} \eta_{1,3} & Z^{T} \eta_{1,4}
\end{array}\right)
$$

where $\eta_{1,1}, \eta_{1,2}, \ldots, \eta_{1,5}$ are given in Appendix B. The scalar $\chi$ in (8) is here chosen in such a way that $\theta_{o}$ belongs to $U_{\hat{\theta}_{N, 1}}$ with a probability of $95 \%$. The largest acceptable worst case performance is specified as $\gamma_{\max }(\omega)=$ 1.05, $\forall \omega$, and we will now use Theorem 2 to validate whether this requirement is fulfilled with the controller $C_{1}$ or not. The computed upper bound of the worst case performance is plotted in Figure 2 for a frequency grid of 100 points. It is clearly larger than our specified $\gamma_{\max }(\omega)$. The controller $C_{1}$ is therefore invalidated.

In order to get an idea of the conservatism of this upper bound, a lower bound is calculated using gridding and plotted in Figure 2. It is clear that the lower bound is close to the upper bound. Thus in this case the conservatism is small.

Since the controller $C_{1}$ is deemed invalidated we now perform experiment design for $\Phi_{r}(\omega)$. The new designed identification experiment will deliver a model $G\left(\hat{\theta}_{N, 2}\right)$ and a model based controller $C_{2}=C_{2}\left(\hat{\theta}_{N, 2}\right)$ which guarantees the worst case performance $\gamma_{\max }(\omega)$. We still consider closed loop identification with $C_{i d}=C_{\text {start }}$ in the loop. This controller $C_{\text {start }}$ is also used as initial estimate for $C\left(\hat{\theta}_{N, 2}\right)$ in (45). The design is based on knowledge on $\theta_{o}$, cf Section 3. Furthermore, we have chosen $m=9$ in (44). The choice of $A_{0}(\omega)$ is improved through $2 \mathrm{D}-\mathrm{K}$ like iterations, which decreases the cost function by $32 \%$. The spectrum $\Phi_{r}(\omega)$ designed after these 2 iterations is shown in Figure 3. A new parameter estimate

\footnotetext{
${ }^{6}$ We have used MATLAB@Version 7.3.0.267 (R2006b) with Control System Toolbox.
}

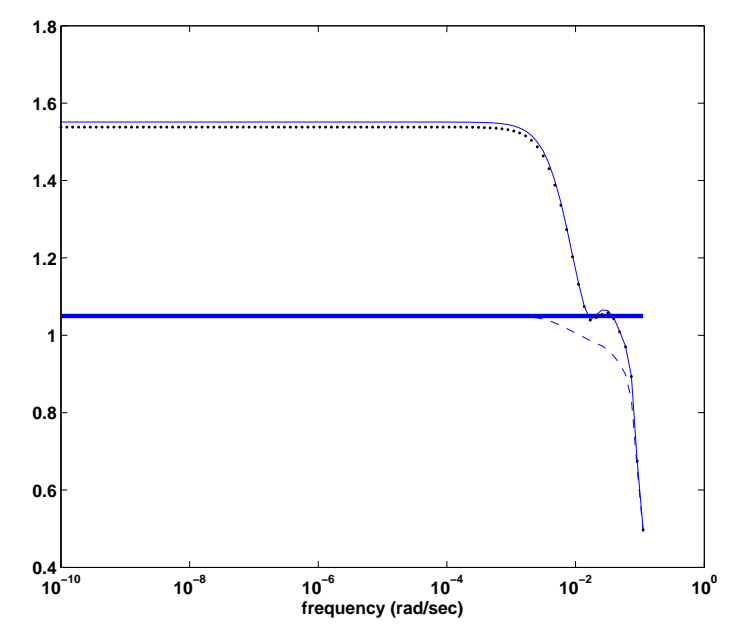

Fig. 2. Thick solid line: $\gamma_{\max }$ versus frequency. Thin solid line: upper bound for the worst case performance $\gamma$ using the controller $C_{1}$. Dotted line: lower bound for $\gamma$ using the controller $C_{1}$. Dashed line (partly coinciding with the thick solid line): upper bound for the worst case performance using $\mathrm{C}_{2}$.

identified with 1000 data generated with the designed excitation signal $r(t)^{7}$ is given by:

$$
\hat{\theta}_{N, 2}=(-0.7570,0.4527,-0.2087,0.2133,-0.4711)^{T}
$$

and a controller $C_{2}=C_{2}\left(\hat{\theta}_{N, 2}\right)$ is designed (with the same $\mathcal{H}_{\infty}$ method as in the first experiment) as

$$
C_{2}(q)=\frac{1}{Z^{T} \eta_{2,5}}\left(\begin{array}{ll}
Z^{T} \eta_{2,1} & Z^{T} \eta_{2,2} \\
Z^{T} \eta_{2,3} & Z^{T} \eta_{2,4}
\end{array}\right)
$$

where $\eta_{2,1}, \eta_{2,2}, \ldots, \eta_{2,5}$ are given in Appendix B. In Figure 2 the resulting upper bound for the worst case performance achieved by $C_{2}$ over the plants in the uncertainty region identified along with $\hat{\theta}_{N, 2}$ is plotted. For each $\omega$, it is clearly equal to or below $\gamma_{\max }(\omega)$ and therefore $C_{2}$ is validated. Also here, a lower bound for the worst case performance is calculated using gridding. It almost coincides with the upper bound.

\section{Conclusions}

This paper presents a new controller validation method for multivariable models obtained with standard PE identification methods. The confidence regions of such models are nonstandard in classical robust control theory. The main contribution is a parametrization of a certain set of multipliers which allows a classical robust

\footnotetext{
7 There are many possible realizations corresponding to one spectrum, see e.g. [11]. Here the signal $r(t)$ is realized as filtered white noise.
} 


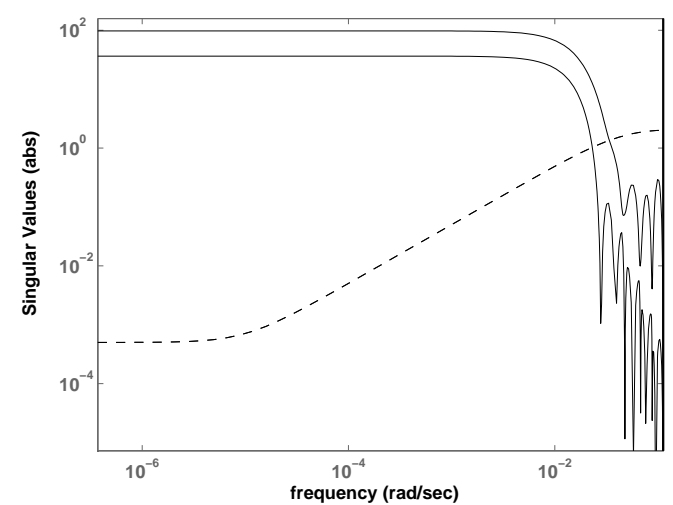

Fig. 3. Solid line: singular values of $\Phi_{r}(\omega)$. Dashed line: $1 / W_{S}$.

control theory framework to be used for the considered confidence regions. Therefore, this paper is part of the wide-spread effort to connect PE system identification and robust control. Only variance errors are considered (i.e. not bias errors) and it is assumed that the data length is large. It is shown that the problem of computing an upper bound for the worst case performance (measured in terms of the $\mathcal{H}_{\infty}$-norm of a weighted closed loop transfer matrix) achieved over all plants in the identified confidence region can be formulated as an LMI-based optimization problem. The method proposed can also be extended to experiment design. More specifically, the method enables us to design the spectrum or the duration of an external excitation signal such that a specified worst case performance is guaranteed. The design is suboptimal, however it can be improved by iterative procedures.

\section{Acknowledgements}

The authors would like to thank the two anonymous referees. Their comments have lead to substantial improvements of the paper.

\section{A Proof of Proposition 3}

Define $\Psi(t, \theta)=\frac{d}{d \theta} \hat{y}(t, \theta)$ and denote the columns of the matrix $\Psi^{T}(t, \theta)$ by $\Psi_{i}^{T}(t, \theta) \in \mathbb{R}^{n_{y}}, i=1, \ldots, n$. With (6)-(7) in (5) we obtain

$$
\Psi_{i}^{T}\left(t, \theta_{o}\right)=F_{r}^{i}\left(q, \theta_{o}\right) r(t)+F_{e}^{i}\left(q, \theta_{o}\right) e(t)
$$

Now, the transpose of (A.1) can be written

$$
\begin{aligned}
\Psi_{i}\left(t, \theta_{o}\right) & =\operatorname{vec}\left(F_{r}^{i}\left(q, \theta_{o}\right)\right)\left(I_{n_{y}} \otimes r(t)\right)+ \\
& +\operatorname{vec}\left(F_{e}^{i}\left(q, \theta_{o}\right)\right)\left(I_{n_{y}} \otimes e(t)\right),
\end{aligned}
$$

which is the $i$ :th row of $\Psi\left(t, \theta_{o}\right)$. This implies that

$$
\begin{aligned}
\Psi\left(t, \theta_{o}\right) & =\left(\begin{array}{c}
\Psi_{1}\left(t, \theta_{o}\right) \\
\Psi_{2}\left(t, \theta_{o}\right) \\
\vdots \\
\Psi_{n}\left(t, \theta_{o}\right)
\end{array}\right)= \\
& =\Gamma_{r}\left(q, \theta_{o}\right)\left(I_{n_{y}} \otimes r(t)\right)+\Gamma_{e}\left(q, \theta_{o}\right)\left(I_{n_{y}} \otimes e(t)\right) .
\end{aligned}
$$

A similar open loop expression for $\Psi\left(t, \theta_{o}\right)$ is found in [24]. When $N \rightarrow \infty$ and the model structure is flexible enough to capture the true system, we have

$$
P^{-1}\left(\theta_{o}\right)=\mathrm{E} \Psi\left(t, \theta_{o}\right) \Lambda_{o}^{-1} \Psi^{T}\left(t, \theta_{o}\right)
$$

[13]. Expressions (37) and (38) are obtained by inserting (A.3) in (A.4), which concludes the proof.

\section{B Controller Parameters}

$$
\begin{aligned}
& \eta_{s, 1}=(1.263,2.585,-0.6939,-4.057,-1.304,1.472,0.7345)^{T} \\
& \eta_{s, 2}=(-0.6145,-1.213,0.3533,1.908,0.611,-0.6947,-0.3502)^{T} \\
& \eta_{s, 3}=(1,1.222,-1.559,-2.467,0.1467,1.244,0.4147)^{T} \\
& \eta_{1,1}=(1.406,2.89,-0.7027,-4.442,-1.49,1.554,0.7872)^{T} \\
& \eta_{1,2}=(-0.5181,-1.025,0.2733,1.578,0.528,-0.5534,-0.2837)^{T} \\
& \eta_{1,3}=(-0.5181,-1.025,0.2733,1.578,0.528,-0.5534,-0.2837)^{T} \\
& \eta_{1,4}=(-1.195,-2.468,0.592,3.793,1.273,-1.326,-0.6708)^{T} \\
& \eta_{1,5}=(1,1.215,-1.562,-2.455,0.1525,1.238,0.4115)^{T} \\
& \eta_{2,1}=(1.238,2.553,-0.6392,-3.97,-1.314,1.418,0.7155)^{T} \\
& \eta_{2,2}=(-0.6064,-1.206,0.3302,1.879,0.6189,-0.6729,-0.3431)^{T} \\
& \eta_{2,3}=(0.5927,1.178,-0.3232,-1.835,-0.6044,0.6572,0.3352)^{T} \\
& \eta_{2,4}=(-1.241,-2.558,0.6407,3.979,1.316,-1.421,-0.7171)^{T} \\
& \eta_{2,5}=(1,1.227,-1.557,-2.478,0.1419,1.249,0.4174)^{T}
\end{aligned}
$$

\section{References}

[1] M. Barenthin, X. Bombois, and H. Hjalmarsson. Mixed $\mathcal{H}_{\infty}$ and $\mathcal{H}_{2}$ input design for multivariable systems. In Proceedings of the 14th IFAC Symposium on System Identification, Newcastle, Australia, March 2006.

[2] M. Barenthin and H. Hjalmarsson. Identification and control: Joint input design and $\mathcal{H}_{\infty}$ state feedback with ellipsoidal parametric uncertainty via LMIs. Automatica, 44(2):543551, February 2008.

[3] X. Bombois and P. Date. Connecting PE identification and robust control theory: the multiple-input single-output case. Part ii: controller validation. In Proceedings of the 13th IFAC Symposium on System Identification, Rotterdam, The Netherlands, 2003. 
[4] X. Bombois, M. Gevers, G. Scorletti, and B.D.O. Anderson. Robustness analysis tools for an uncertainty set obtained by prediction error identification. Automatica, 37(10):16291636, 2001.

[5] X. Bombois, G. Scorletti, M. Gevers, P. Van den Hof, and R. Hildebrand. Least costly identification experiment for control. Automatica, 42(10):1651-1662, October 2006.

[6] B.L. Cooley and J. H. Lee. Control-relevant experiment design for multivariable systems described by expansions in orthonormal bases. Automatica, 37:273-281, 2001.

[7] L. Gerencsér and H. Hjalmarsson. Adaptive input design in system identification. In Proceedings of the 44th IEEE Conference on Decision and Control, Seville, Spain, December 2005.

[8] K-C. Goh and M.G. Safonov. Robust analysis, sectors, and quadratic functionals. In Proceedings of the 34th IEEE Conference on Decision and Control, New Orleans, LA, USA, 1995.

[9] G. C. Goodwin and R. L. Payne. Dynamic System Identification: Experiment Design and Data Analysis. Academic Press, New York, 1977.

[10] R. Hildebrand and M. Gevers. Identification for control: Optimal input design with respect to a worst case $\nu$-gap cost function. SIAM Journal on Control and Optimization, 41(5):1586-1608, 2003.

[11] H. Jansson and H. Hjalmarsson. Input design via LMIs admitting frequency-wise model specifications in confidence regions. IEEE Transactions on Automatic Control, 50(10):1534 - 1549, October 2005.

[12] K. Lindqvist and H. Hjalmarsson. Identification for control: Adaptive input design using convex optimization. In Proceedings of the 40th IEEE Conference on Decision and Control, Orlando, US, December 2001.

[13] L. Ljung. System Identification - Theory For the User, 2nd ed. Prentice Hall, Upper Saddle River, New Jersey, 1999.

[14] R. Mehra. Optimal input signals for parameter estimation in dynamic systems - survey and new results. IEEE Transactions on Automatic Control, 19(6):753-768, December 1974.

[15] M.G. Safonov. Stability and Robustness of Multivariable Feedback Systems. MIT Press, Cambridge, MA, 1980.

[16] G. Scorletti. Robustness analysis with time-delays. In Proceedings of the 36th IEEE Conference on Decision and Control, pages 3824-3829, San Diego, US, December 1997.

[17] G. Scorletti, X. Bombois, M. Barenthin, and V. Fromion. Improved efficient analysis for systems with uncertain parameters. In Proceedings of the 46th IEEE Conference on Decision and Control, New Orleans, USA, December 2007.

[18] S. Skogestad and I. Postlethwaite. Multivariable feedback control-analysis and design ( $2^{\text {nd }}$ edition). Wiley, Chippenham, Wiltshire, 2005.

[19] T. Söderström and P. Stoica. System identification. Prentice Hall International, Hertfordshire, UK, 1989.

[20] B. Wahlberg. System identification using Laguerre models. IEEE Transactions on Automatic Control, 36(5):551-562, 1991.

[21] S.P. Wu, S. Boyd, and L. Vandenberghe. FIR filter design via semidefinite programming and spectral factorization. In Proceedings of the 35th IEEE Conference on Decision and Control, Kobe, Japan, December 1996.

[22] V.A. Yakubovich. Solution of certain matrix inequalities occurring in the theory of automatic control. Docl. Acad. Nauk. SSSR, pages 1304-1307, 1962.

[23] K. Zhou, J. Doyle, and K. Glover. Robust and optimal control. Prentice Hall, Upper Saddle River, New Jersey, 1996.
[24] Y.C. Zhu. Black-box identification of MIMO transfer functions: asymptotic properties of prediction error models. Int $J$ of Adaptive Control and Signal Processing, 3:357-373, 1989 . 